\title{
ACTH stimulation test and computed tomography are useful for differentiating the subtype of primary aldosteronism
}

\author{
Ayako Moriya $^{1)}$, Masaaki Yamamoto ${ }^{1)}$, Shunsuke Kobayashi ${ }^{1)}$, Tomoko Nagamine ${ }^{1)}$, \\ Naomi Takeichi-Hattori ${ }^{1)}$, Mototsugu Nagao ${ }^{1)}$, Taro Harada ${ }^{1)}$, Kyoko Tanimura-Inagaki ${ }^{1)}$, \\ Shiro Onozawa ${ }^{2)}$, Satoru Murata ${ }^{2)}$, Hideki Tamura ${ }^{1), 3)}$, Izumi Fukuda ${ }^{1)}$, Shinichi Oikawa ${ }^{1), 4)}$ and \\ Hitoshi Sugihara ${ }^{1)}$ \\ 1) Department of Endocrinology, Diabetes and Metabolism, Graduate School of Medicine, Nippon Medical School, Tokyo \\ 113-8603, Japan \\ 2) Department of Radiology/Center for Advanced Medical Technology, Nippon Medical School, Tokyo 113-8603, Japan \\ 3) Tamura Medical Clinic, Tokyo 132-0013, Japan \\ 4) Department of Diabetes and Lifestyle Disease Center, Fukujuji Hospital, Japan Anti-Tuberculosis Association (JATA), Tokyo \\ 204-8522, Japan
}

\begin{abstract}
The diagnostic steps for primary aldosteronism (PA) include case screening tests, confirmatory tests, and localization. The aim of this study was to identify useful confirmatory tests and their cut-off values for differentiating the subtype of primary aldosteronism, especially in unilateral PA, such as aldosterone-producing adenoma, and bilateral PA, such as idiopathic hyperaldosteronism. Seventy-six patients who underwent all four confirmatory tests, the captopril-challenge test (CCT), furosemide upright test (FUT), saline infusion test (SIT), and ACTH stimulation test (AST), and who were confirmed to have an aldosterone excess by adrenal venous sampling (AVS) were recruited. Subjects were diagnosed as having unilateral aldosterone excess $(n=17)$ or bilateral aldosterone excess $(n=59)$ by AVS. The SIT-positive rate was significantly higher in the unilateral group (94.1\%) than in the bilateral group (57.6\%). Multivariable logistic regression analysis showed that tumor on computed tomography (CT) and plasma aldosterone concentration $(\mathrm{PAC})_{\max } /$ cortisol on the AST were useful for differentiating the subtype of PA. Receiver operating characteristic (ROC) curve analysis for distinguishing the subtype of PA showed that a cut-off value of 18.3 $\mathrm{PAC}_{\max } /$ cortisol on the AST had a sensitivity of $83 \%$ and a specificity of $88 \%$. The area under the ROC curve was 0.918 (95\% confidence interval 0.7916-0.9708). These data suggest that abdominal CT and AST are useful for differentiating the subtype of PA and the indication for AVS.
\end{abstract}

Key words: Primary aldosteronism, Subtype, ACTH stimulation test, Computed tomography, Saline infusion test

PRIMARY ALDOSTERONISM (PA) is the most important cause of secondary hypertension and is associated with a higher risk of cardiovascular events and renal damage than essential hypertension [1-3]. It has been shown that aldosterone excess may be associated with endothelial dysfunction independent of blood pressure and produce microvascular inflammation not only in the brain but also in the myocardium [4]. Furthermore, recent experimental studies

Submitted Jun. 8, 2016; Accepted Aug. 29, 2016 as EJ16-0297 Released online in J-STAGE as advance publication Oct. 1, 2016 Correspondence to: Izumi Fukuda, Department of Endocrinology, Diabetes and Metabolism, Graduate School of Medicine, Nippon Medical School, 1-1-5 Sendagi, Bunkyo-ku, Tokyo 113-8603, Japan. E-mail: i-fukuda@nms.ac.jp

(c) The Japan Endocrine Society have shown that chronic aldosterone excess produces increased aortic stiffness, independent of blood pressure [5]. Therefore, the early diagnosis and treatment of PA are important to prevent vascular target organ damage caused by aldosterone excess. In epidemiological studies, the incidence of PA in hypertensive patients ranged widely, from $3.2 \%$ to $21.7 \%$ [6]. The difference in the prevalence of PA among various studies is mainly caused by the different cut-off values for the screening or confirmatory tests. The diagnostic guidelines for PA of the Japan Endocrine Society (JES) recommend measuring the plasma renin activity (PRA) $(\mathrm{ng} / \mathrm{mL} / \mathrm{h})$ and plasma aldosterone concentration $(\mathrm{PAC})(\mathrm{pg} / \mathrm{mL})$ and then calculating the PAC/PRA 
ratio (ARR) in patients initially diagnosed as having hypertension. If the ARR is over 200, the JES recommends making a definitive diagnosis by performing at least 2 of 3 confirmatory tests, the captopril-challenge test (CCT), the furosemide upright test (FUT), and the saline infusion test (SIT) [7]. On the other hand, the Japanese Society of Hypertension (JSH) recommends that PA should be diagnosed by performing at least 1 confirmatory test [8]. Furthermore, the ACTH stimulation test (AST) is also reported to be useful for diagnosing PA accurately [9].

Once PA is diagnosed, a localized diagnosis is necessary. Aldosterone-producing adenoma (APA) (unilateral) and idiopathic hyperaldosteronism (IHA) (bilateral) are the most common subtypes of primary aldosteronism [10, 11]. Adrenal venous sampling (AVS) is the gold-standard test to differentiate unilateral from bilateral disease, and it should be offered to patients who have been diagnosed with PA based on confirmatory tests and wish to be treated surgically [7, 11]. However, AVS is far from easy to perform for all patients with PA because of some problems (e.g., high cost, adrenal phlebography-associated complications, and the high level of technical skill and experience needed for successful cannulation of the (right) adrenal vein). Recent reports have shown that the ACTH stimulation test with 1-mg dexamethasone is useful for determining the subtype of PA $[12,13]$. However, little has been reported about which confirmatory tests are useful in determining the subtype of PA. Our objective was to determine the most useful confirmatory test and the cut-off value to distinguish the subtype of PA.

\section{Subjects and Methods}

\section{Patients}

The medical records of patients diagnosed with PA by confirmatory testing and localization by AVS were retrospectively analyzed. Ninety patients admitted to Nippon Medical School Hospital for AVS from January 2009 to March 2015 were examined. This was a retrospective study without intervention that was approved by the Nippon Medical School and Faculty of Medicine Ethics Committee and conducted in accordance with the principles of the Declaration of Helsinki. This study was registered with the University Hospital Medical Information Network (UMIN) (No. UMIN000022657). The diagnosis was confirmed by endocrinological examinations such as confirmatory tests (CCT, FUT, and SIT) and ACTH-stimulated AVS (ACTH-AVS). A definitive diagnosis of PA was based on at least one positive confirmatory test (CCT, FUT, or SIT). AST and computed tomography (CT) were performed in all patients.

\section{Assays}

Serum cortisol levels were measured by an enzyme immunoassay (EIA) kit (Tosoh Corporation, Tokyo, Japan). PAC and PRA were measured by radioimmunoassay (Fujirebio Inc., Tokyo, Japan).

\section{Procedure of diagnosis in $P A$}

Hypertensive patients with ARR over 200 were targeted $[7,14]$. All antihypertensive drugs except calcium channel blockers and $\alpha$-blockers were stopped at least 2 weeks before the examination [7]. Plasma potassium concentrations of $3.5 \mathrm{mEq} / \mathrm{L}$ or less were corrected by oral supplementation. All tests were performed during morning hours in a quiet room. In particular, PRA and PAC were measured in blood samples obtained after $30 \mathrm{~min}$ of rest in a supine position in the morning.

\section{Confirmatory tests}

CCT, FUT, and SIT were performed after overnight fasting according to the JES guidelines [7].

\section{CCT}

Patients took captopril $50 \mathrm{mg}$ (crushed orally). Blood samples were obtained before and after taking captopril. ARR over 200 at 60 minutes after captopril administration was considered positive.

FUT

Patients were administered $40 \mathrm{mg}$ of furosemide by an intravenous injection and maintained in a standing position for two hours. PRA values less than $2.0 \mathrm{ng} /$ $\mathrm{mL} / \mathrm{h}$ at 60 and 120 minutes after loading were considered positive.

\section{SIT}

Patients were infused with $2 \mathrm{~L}$ of $0.9 \%$ saline over 4 hours. The test was considered positive when PAC was higher than $60 \mathrm{pg} / \mathrm{mL}$ after the saline infusion.

AST

Patients were administered $250 \mu \mathrm{g}$ of synthetic ACTH (tetracosactide acetate: Cortrosyn ${ }^{\circledR}$ (Daiichi Sankyo Company, Limited, Tokyo, Japan)) by intravenous injection. Blood samples were obtained before and every 30 minutes for 2 hours after ACTH injec- 
tion. A ratio of PAC to cortisol (F) over 8.5 at the time of peak concentration of aldosterone after ACTH injection $\left(\mathrm{PAC}_{\max } / \mathrm{F}\right)$ was considered positive [9].

\section{CT scan}

All but one patient underwent $\mathrm{CT}$ of the adrenal glands with contiguous $0.625,1.25,2.5$, or 5 -mm slice cuts. Only one patient underwent a $10-\mathrm{mm}$ scan in another facility. All scans were reviewed by several experienced radiologists. An adrenal tumor on CT was defined as the presence of a solitary or clearly delineated nodule; "mild adrenal enlargement" and "a questionable nodule" were regarded as no nodule.

\section{Adrenal venous sampling}

Expert radiologists performed AVS. To confirm that the catheter tip was inserted into the adrenal vein, $\mathrm{CT}$ venography with contrast media was performed [15]. After confirming that the catheter tip was inserted into the adrenal vein, blood samples were collected, and tetracosactide acetate (synthetic ACTH) was injected intravenously. Blood samples were obtained within 15 to 45 minutes after ACTH stimulation [15]. The criteria for adequate catheterization into the adrenal vein and aldosterone hypersecretion were according to the JES guidelines [7]. An adrenal venous cortisol concentration after ACTH stimulation over 200 $\mu \mathrm{g} / \mathrm{dL}$ was considered evidence of adequate catheterization for AVS. Aldosterone hypersecretion was considered present when PAC in the adrenal vein after ACTH stimulation was over $14,000 \mathrm{pg} / \mathrm{mL}$. If both adrenal venous PAC levels were over $14,000 \mathrm{pg} / \mathrm{mL}$, a bilateral lesion, such as IHA, was considered likely. On the other hand, when adrenal venous PAC was over $14,000 \mathrm{pg} / \mathrm{mL}$ on only one side, a unilateral lesion was considered likely. Furthermore, the adrenal venous aldosterone/cortisol (A/C) ratio after ACTH stimulation was calculated bilaterally, and when the lateralized ratio (adrenal vein $\mathrm{A} / \mathrm{C}$ ratio on the high-value side)/ (adrenal vein $\mathrm{A} / \mathrm{C}$ ratio on the low-value side) was over 2.6, a unilateral lesion was considered present on the high value side. Since aldosterone production in the contralateral normal adrenal gland is supposed to be suppressed in typical unilateral disease, a unilateral lesion was considered when the contralateral ratio (adrenal vein $\mathrm{A} / \mathrm{C}$ ratio on the low-value side)/(A/C ratio in the inferior vena cava) was less than 1.0. A unilateral lesion was finally diagnosed only when PAC was over $14,000 \mathrm{pg} / \mathrm{mL}$ on one side, the lateralized ratio was over 2.6, and the contralateral ratio was less than $1.0[7,16]$.

\section{Exclusion criteria}

The following patients were excluded from this study: one patient who had iodine allergy and used steroid during AVS; five patients who were diagnosed with subclinical Cushing's syndrome; four patients taking antihypertensive drugs other than calcium channel blockers and $\alpha$-blockers for refractory hypertension; one patient with bilateral aldosterone-producing adenomas, confirmed by the clinical course and pathology after surgery (left adrenal gland tumor segmental resection and right adrenalectomy); two patients with unsuccessful AVS; and one patient who had unilateral aldosterone excess (PAC > $14,000 \mathrm{pg} / \mathrm{mL}$ ) and LR over 2.6, but CR over 1.0 on AVS. The data of 76 of the 90 initial patients were ultimately analyzed.

\section{Statistical analysis}

Fisher's exact test was used for categorical variables, and the Wilcoxon rank-sum test was used for continuous variables to assess differences in clinical data between unilateral and bilateral primary aldosteronism. Fisher's exact test was used to identify differences in the positive rate of each confirmatory test, and the Cochran-Armitage trend test was used to investigate the trend of the number of positive confirmatory tests between unilateral and bilateral aldosterone excess. Each value that had significant associations with bilateral aldosterone excess on univariate analysis was then taken forward to multivariate models. Stepwise selection was performed to find variables independently associated with localization. Entry and removal probabilities for stepwise selection were both 0.05 . A receiver operating characteristic (ROC) curve was generated, and the area under the curve was calculated to determine the best level for determining the subtype.

All statistical analyses were carried out using JMP version 11 (SAS Institute Inc., Cary, NC). A $P$ value $<$ 0.05 was considered significant.

\section{Results}

\section{Patient characteristics}

A total of 76 patients were included in the analysis. In all of these patients, aldosterone hypersecretion 
from unilateral or bilateral adrenal glands was confirmed by ACTH-AVS. Patient characteristics are shown in Table 1. Patients were divided into two groups: unilateral aldosterone hypersecretion (unilateral group, $\mathrm{n}=17$; men 9) and bilateral aldosterone hypersecretion (bilateral group, $\mathrm{n}=59$; men 22). PAC (unilateral group, $444.1 \pm 282.1 \mathrm{pg} / \mathrm{mL}$; bilateral group, $118.2 \pm 41.3 \mathrm{pg} / \mathrm{mL} ; p<0.0001$ ) and ARR (unilateral group, 3,321.4 $\pm 2,908.3$; bilateral group, 484.9 $\pm 338.8 ; p<0.0001$ ) were significantly higher in the unilateral group than in the bilateral group. The serum potassium level was significantly lower in the unilateral group than in the bilateral group (unilateral group, $3.02 \pm 0.71 \mathrm{mEq} / \mathrm{L}$; bilateral group, $4.01 \pm 0.37 \mathrm{mEq} / \mathrm{L}$; $p<0.0001$ ), as was PRA (unilateral group, $0.18 \pm$ $0.10 \mathrm{ng} / \mathrm{mL} / \mathrm{h}$; bilateral group, $0.33 \pm 0.18 \mathrm{ng} / \mathrm{mL} / \mathrm{h}$; $p<0.001)$. In the unilateral group, 10 cases took oral potassium supplementation for hypokalemia, while no cases in the bilateral group required potassium supplementation. An adrenal tumor was seen on CT scan in 14 of 17 cases in the unilateral group and in 11 of 59 cases in the bilateral group; the percentage was significantly lower in the bilateral group $(p<0.0001)$. Tumor size was $19.0 \pm 10.4-\mathrm{mm}$ in the unilateral group and $13.0 \pm 4.3-\mathrm{mm}$ in the bilateral group. In the unilateral group, adrenal tumors were not found in three patients (including one patient with a questionable nodule). In the bilateral group, adrenal tumors were not found in 48 patients (including three patients with questionable nodules and two patients with mild adrenal enlarge- ment), and one patient had bilateral tumors. In the unilateral group, tumor localization on $\mathrm{CT}$ was consistent with the results of AVS in all cases.

\section{Positive confirmatory tests}

The percentages of patients with positive CCT, FUT, SIT, and AST results were $84.2 \%, 85.5 \%$, $65.8 \%$, and $93.4 \%$, respectively (Table 2 ). There was a significant difference in the SIT-positive rate between the unilateral group and the bilateral group (unilateral group, 94.1\%; bilateral group, 57.6\%; $p<$ 0.01 ), whereas there were no significant differences in the positive rate between the two groups for the CCT (unilateral group, 94.1\%; bilateral group, $81.4 \% ; p=$ 0.28 ), FUT (unilateral group, $100 \%$; bilateral group, $81.4 \% ; p=0.11$ ), and AST (unilateral group, 94.1\%; bilateral group, $93.2 \% ; p=1.00$ ).

\section{Number of positive tests}

The number of positive tests in each case is shown in Table 3. All four confirmatory tests were positive in 15 of 17 cases in the unilateral group; in the other two unilateral group cases, three tests except AST were positive in one case, and in the other case, only FUT and AST were positive. In the bilateral group, all confirmatory tests were positive in 24 of 59 cases; one case was positive only on the CCT. These data showed that the number of positive tests had a significant trend toward increasing in the unilateral group compared to the bilateral group on the Cochran-Armitage trend test

Table 1 Patient characteristics

\begin{tabular}{lccc}
\hline & Unilateral PA & Bilateral PA & $P$ \\
\hline $\mathrm{n}$ & 17 & 59 & $0.27^{\mathrm{b}}$ \\
Male/Female & $9 / 8$ & $22 / 37$ & $0.06^{\mathrm{a}}$ \\
Age $(\mathrm{y})$ & $46.4 \pm 12.9$ & $53.5 \pm 12.1$ & $0.25^{\mathrm{a}^{\mathrm{a}}}$ \\
Number of antihypertensive drugs & $1.00 \pm 0.61$ & $0.81 \pm 0.54$ & $0.90^{\mathrm{a}}$ \\
Systolic BP $(\mathrm{mmHg})$ & $137.3 \pm 14.6$ & $137.9 \pm 16.5$ & $0.45^{\mathrm{a}}$ \\
Diastolic BP $(\mathrm{mmHg})$ & $75.7 \pm 7.92$ & $78.6 \pm 11.1$ & $<0.0001^{\mathrm{a}}$ \\
Serum potassium $(\mathrm{mEq} / \mathrm{L})$ & $3.02 \pm 0.71$ & $4.01 \pm 0.37$ & $<0.0001^{\mathrm{a}}$ \\
PAC $(\mathrm{pg} / \mathrm{mL})$ & $444.1 \pm 282.1$ & $118.2 \pm 41.3$ & $<0.001^{\mathrm{a}}$ \\
PRA $(\mathrm{ng} / \mathrm{mL} / \mathrm{h})$ & $0.18 \pm 0.10$ & $0.33 \pm 0.18$ & $<0.0001^{\mathrm{a}}$ \\
ARR & $3321.4 \pm 2908.3$ & $484.9 \pm 338.8$ & $<0.0001^{\mathrm{b}}$ \\
Adrenal tumor on CT(\%) & $14 / 17(82.3)$ & $11 / 59(18.6)$ & $0.001^{\mathrm{a}}$ \\
Number of positive tests & $3.82 \pm 0.53$ & $3.12 \pm 0.85$ & $<0.0001^{\mathrm{a}}$ \\
PAC max $/$ cortisol on AST & $40.6 \pm 23.9$ & $14.4 \pm 5.25$ & $<0.0001^{\mathrm{a}}$ \\
PAC/PRA at 60 min on CCT & $2839.2 \pm 2390.0$ & $370.9 \pm 349.9$ & $<0.001^{\mathrm{a}}$ \\
PRA at 120 min on FUT & $0.42 \pm 0.30$ & $1.24 \pm 1.28$ & $<0.0001^{\mathrm{a}}$ \\
PAC at 240 min on SIT & $452.8 \pm 363.2$ & $67.4 \pm 31.6$ &
\end{tabular}

Data are expressed as means \pm SD. BP, blood pressure; PAC, plasma aldosterone concentration; PRA, plasma renin activity; ARR, aldosterone to renin ratio; AST, ACTH stimulation test; CCT, captopril challenge test; FUT, furosemide upright test; SIT, saline infusion test. ${ }^{\mathrm{a}} P$ value for Wilcoxon rank-sum test, ${ }^{\mathrm{b}} P$ value for Fisher's exact test. 
(unilateral group, $3.82 \pm 0.53$; bilateral group, $3.12 \pm$ $0.85 ; p<0.01)$.

\section{Response to confirmatory test}

The response on each confirmatory test was compared between the bilateral group and the unilateral group (Fig. 1). On the CCT, PAC/PRA was higher in the unilateral group than in the bilateral group before and after the test. On the FUT, the PRA was lower in the unilateral group than in the bilateral group at all times. On the SIT, the PAC was higher in the unilateral group than in the bilateral group at all times. On the $\mathrm{AST}$, the PAC/F was higher in the unilateral group than in the bilateral group at all times.

Table 2 Positive rates for each confirmatory test in patients with unilateral PA and bilateral PA

\begin{tabular}{lcccc}
\hline & Total & Unilateral PA $(\mathrm{n}=17)$ & Bilateral PA $(\mathrm{n}=59)$ & ${ }^{\mathrm{a}} P$ value \\
\hline CCT & $64 / 76(84.2 \%)$ & $16 / 17(94.1 \%)$ & $48 / 59(81.4 \%)$ & 0.28 \\
FUT & $65 / 76(85.5 \%)$ & $17 / 17(100 \%)$ & $48 / 59(81.4 \%)$ & 0.11 \\
SIT & $50 / 76(65.8 \%)$ & $16 / 17(94.1 \%)$ & $34 / 59(57.6 \%)$ & $<0.01$ \\
AST & $71 / 76(93.4 \%)$ & $16 / 17(94.1 \%)$ & $55 / 59(93.2 \%)$ & 1.00 \\
\hline
\end{tabular}

Data are expressed as the positive rate for each confirmatory test. AST, ACTH stimulation test; CCT, captopril challenge test; FUT, furosemide upright test; SIT, saline infusion test. ${ }^{a} P$ value for Fisher's exact test.

Table 3 Number of positive tests including the AST

\begin{tabular}{llccccc}
\hline & 1 & 2 & 3 & 4 & Total & $P$ \\
\hline Unilateral & $0(0 \%)$ & $1(5.9 \%)$ & $1(5.9 \%)$ & $15(88.2 \%)$ & 17 & $<0.01^{\text {a }}$ \\
Bilateral & $1(1.7 \%)$ & $15(25.4 \%)$ & $19(32.2 \%)$ & $24(40.7 \%)$ & 59 & \\
Total & $1(1.3 \%)$ & $16(21.1 \%)$ & $20(26.3 \%)$ & $39(51.3 \%)$ & 76 & \\
\hline
\end{tabular}

${ }^{\mathrm{a}} P<0.01$ vs. Bilateral. $P$ value for Cochran-Armitage trend test.
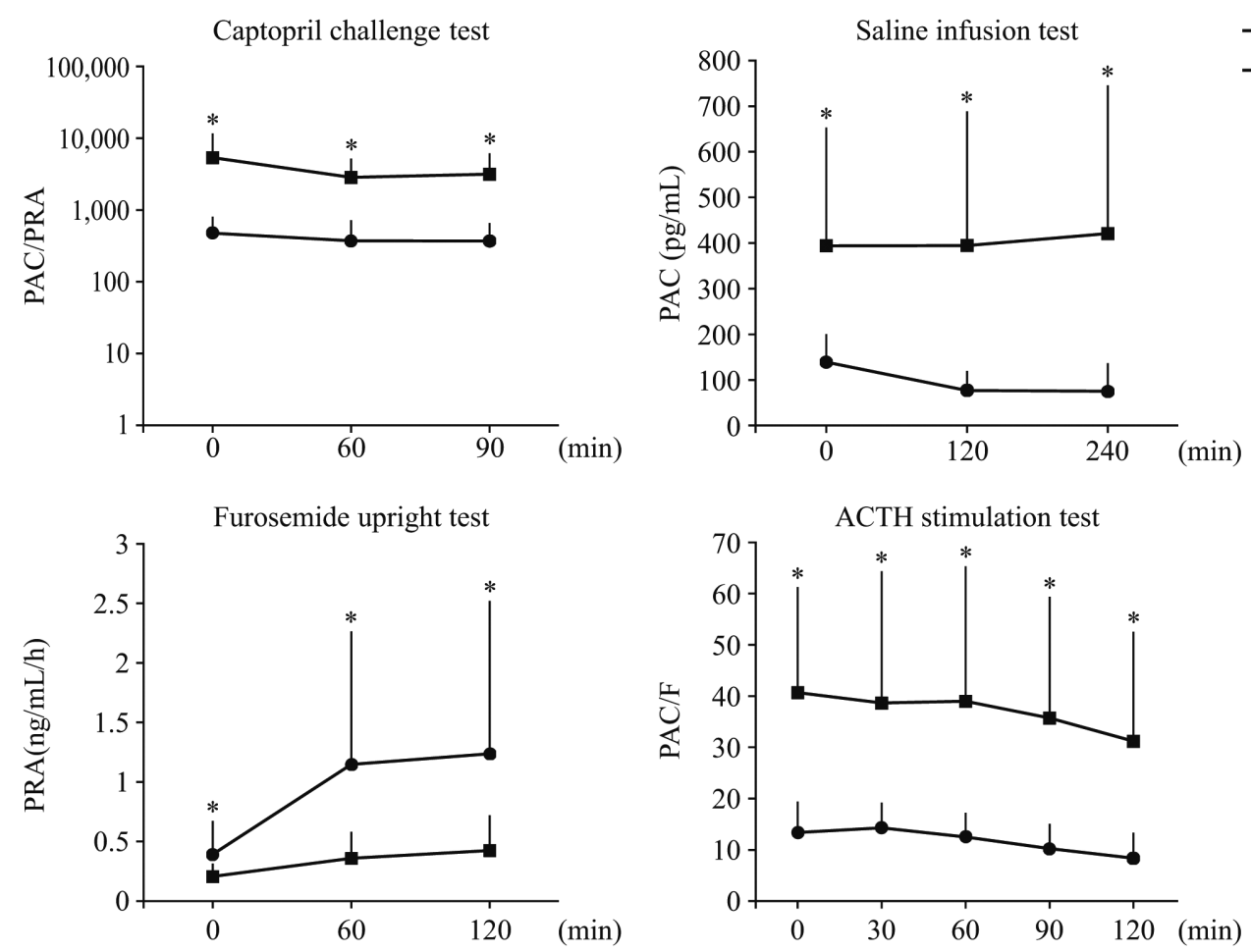

Fig. 1 Results for each confirmatory test in the unilateral and bilateral PA groups

Closed circles indicate the bilateral group and closed squares indicate the unilateral group. Data are expressed as mean $\pm \mathrm{SD}$. As compared with the bilateral group, ${ }^{*}$ indicates $P<0.05$. 


\section{Predictors for the bilateral group}

The results of PA patients who underwent the four confirmatory tests, AVS, and CT were analyzed to identify predictors for localization. Variables associated with lateralization on univariable analysis (serum potassium, PRA, ARR, the presence of tumor on $\mathrm{CT}$, number of positive tests, $\mathrm{PAC}_{\max } / \mathrm{F}$ on $\mathrm{AST}, \mathrm{PAC} / \mathrm{PRA}$ at $60 \mathrm{~min}$ on CCT, PRA at $120 \mathrm{~min}$ on FUT, PAC at 240 min on SIT), age, and sex were entered in the model by stepwise selection. PAC was excluded because of a strong correlation with ARR and PAC at $240 \mathrm{~min}$ on SIT. $\mathrm{PAC}_{\max } / \mathrm{F}$ on AST and the presence of tumor on CT were significant in the regression model (Table 4). The ROC curve of $\mathrm{PAC}_{\max } / \mathrm{F}$ on the AST to diagnose bilateral PA is shown in Fig. 2. When a cut-off value for $\mathrm{PAC}_{\max } / \mathrm{F}$ was set at below 18.3 to diagnose bilateral PA, the sensitivity and specificity were $83 \%$ and $88 \%$, respectively, and the area under the ROC curve (AUC) was 0.918 (95\%CI 0.7916-0.9708).

\section{Discussion}

This study showed that the basal values of PAC and ARR were lower, and serum potassium and PRA levels were higher in the bilateral group than in the unilateral group. Although the positive rate was higher in the unilateral group than in the bilateral group only for the SIT, the number of positive tests had significant trends toward increasing in the unilateral group compared to the bilateral group. The PAC and PRA responses in all confirmatory tests including the AST were different between the unilateral group and the bilateral group. The purpose was to identify predictors of bilateral aldosterone excess cases that do not require surgery, and the regression model showed that $\mathrm{PAC}_{\max } / \mathrm{F}$ on the AST and the presence of tumor on CT were significant factors for localization.

Some scores for predicting unilateral primary aldosterone cases were reported previously [17, 18]. However, the predictive indices differ in each report. One report used a typical Conn's adenoma and at least one of two supplementary biochemical characteristics (serum potassium $<3.5 \mathrm{mmol} / \mathrm{L}$ or estimated glomerular filtration rate $\geq 100 \mathrm{~mL} / \mathrm{min} / 1.73 \mathrm{~m}^{2}$ ) [17]. The other report used the criteria of serum potassium $\leq 3.4 \mathrm{mEq} / \mathrm{L}, \mathrm{PAC} \geq 165 \mathrm{pg} / \mathrm{mL}$, and $\mathrm{ARR} \geq 1,000$ on the CCT [18]. Minami et al. reported that some PA patients, if not all, with a distinct unilateral adrenal lesion on CT and profound hypokalemia, younger
Table 4 Coefficients of variables entered into the multivariable logistic regression models

\begin{tabular}{lccc}
\hline Variable & Coefficient & $P$-value & $95 \%$ CI \\
\hline AST $P A C_{\max } / \mathrm{F}$ & -0.20 & 0.0027 & -0.364 to -0.091 \\
No tumor on CT & 1.36 & 0.0061 & 0.460 to 2.489 \\
\hline
\end{tabular}

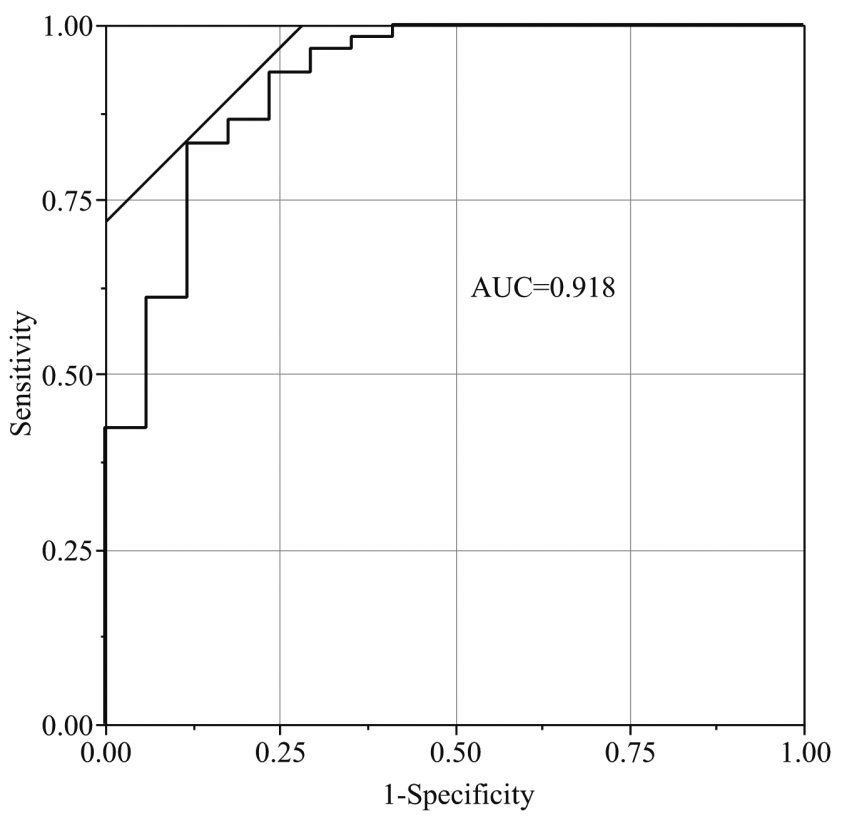

Fig. 2 ROC curve of $\mathrm{PAC}_{\max } /$ cortisol in the ACTH stimulation test

ROC curve analysis of the ability of each variable to predict a bilateral lesion. $\mathrm{PAC}_{\max } / \mathrm{F}$ less than $18.3 \mathrm{pg} /$ $\mathrm{mL}$ has a sensitivity of $83 \%$ and specificity of $88 \%$, with AUC of 0.918 . $\mathrm{PAC}_{\max } / \mathrm{F}$, ratio of peak concentration of aldosterone after ACTH to cortisol; PAC, plasma aldosterone concentration; AUC, area under the ROC curve.

age, and poor response of the PAC after FUT, may not require AVS in the PA subgroup [19]. In the present study, potassium concentration showed a trend to be lower in the unilateral group than in the bilateral group, but potassium concentration was not identified as a predictive factor on multivariate analysis. Since there were cases who had already taken potassium preparations for hypokalemia, this was considered one of the reasons why potassium concentration was not a predictive factor in this study.

The present study showed significant differences in the SIT-positive rate between the unilateral group and the bilateral group. On the other hand, a previous report showed that SIT failed to distinguish unilateral from bilateral lesions, while SIT showed high 
diagnostic accuracy in PA [20]. This discrepancy in the response to SIT might be caused by differences between races or in salt intake.

The AST has not yet been established as a confirmatory test, and there is room for discussion about the usefulness of the AST for diagnosing PA. However, the AST has spread widely in Japan because of its convenience and safety. The present data showed that the AST had the highest positive rate among all confirmatory tests in both the unilateral and bilateral groups. Sonoyama et al. and Jiang et al. reported that the ACTH stimulation test under dexamethasone suppression is highly sensitive for distinguishing APA from IHA or non-PA $[12,13]$. In this study, the AST-positive rate was not different between the groups using a cut-off value of 8.5 for $\mathrm{PAC}_{\max } / \mathrm{F}$ in AST [9]. However, the mean $\mathrm{PAC}_{\max } / \mathrm{F}$ values of the unilateral group and bilateral group were 40.6 and 14.4, respectively, and the difference was significant $(P<0.0001)$. ROC curve analysis confirmed 18.3 as the best diagnostic cut-off value of $\mathrm{PAC}_{\max } / \mathrm{F}$ on the $\mathrm{AST}$ for distinguishing the subtype of PA, suggesting that a unilateral group, such as with APA, was more responsive to ACTH stimulation than a bilateral group, such as with IHA, as described previously [21]. Omura et al. showed that PAC was significantly higher in APmacroA ( $\geq 7 \mathrm{~mm})$ than in APmicroA (less than $6 \mathrm{~mm}$ ) [22]. In the present series, $82 \%$ of the patients in the unilateral group $(14 / 17)$ had a macro adenoma and high PAC. These characteristics of the patients in the unilateral group might cause clearly elevated PAC and PAC/F at baseline in comparison with other studies [12, 22, 23]. The remarkably high $\mathrm{PAC} / \mathrm{F}$ in the unilateral group persisted until $120 \mathrm{~min}$ after ACTH administration, and it might have led to the clinical usefulness of AST in differentiating the subtype of PA. Terui et al. reported that $\mathrm{PAC}_{\max }$ was more useful than $\mathrm{PAC}_{\max } / \mathrm{F}$ in AST without dexamethasone suppression for differentiating between APA patients and other patients [23]. In the present study, the AUC of $\mathrm{PAC}_{\max }$ for differentiating between the unilateral group and the bilateral group was 0.864 (95\% CI 0.6941-0.9471), and the optimal cut-off value of $\mathrm{PAC}_{\max }$ was $>605.7 \mathrm{pg} / \mathrm{mL}$. The sensitivity and specificity were $98 \%$ and $65 \%$, respectively, suggesting that the $\mathrm{PAC}_{\max }$ had lower specificity than $\mathrm{PAC}_{\max } / \mathrm{F}$ (sensitivity $83 \%$ and specificity $88 \%$ ). Terui et al. classified patients into three groups, and the probable PA included 40 patients who did not undergo AVS. In the present series, all patients were classified based on the results of AVS. There is a possibility that the different classification of patients caused discordant results.

A previous report showed that, while the post-FUT PRA was lower in APA than in non-APA, ARR was more accurate than the post-FUT PRA in screening for APA [24]. This study also showed that the post-FUT PRA was not meaningful for differentiating localization on bivariate logistic regression analysis. Thus, this study suggests that FUT is not useful in differentiating the subtype of PA.

In the present study, the presence of tumor on CT was a significant factor for localization in the regression model. However, in a systemic review, Kempers et al. reported a strikingly high rate (37.8\%) of CT/ MRI results that were not consistent with AVS results. Assuming that AVS is $100 \%$ accurate in detecting lateralization of aldosterone secretion, the hypothetical sole reliance on CT/MRI to determine treatment strategy would have resulted in inappropriate adrenalectomy in $14.6 \%$ of patients, inappropriate exclusion from adrenalectomy in $19.1 \%$, and adrenalectomy on the inappropriate side in $3.9 \%$. In detail, a unilateral lesion on AVS with bilateral lesions or normal adrenal glands on CT or MRI was seen in 181/950 (19.1\%) cases [25]. In this study, most patients had a macroadenoma rather than microadenoma, and only two patients in the bilateral group but no patient in the unilateral group had tumor less than $10-\mathrm{mm}$ in diameter. Taking these findings into consideration, the discrepancy in the usefulness of CT findings as a predictor of localization between this study and previous report by Kempers et al. might be caused by different background factor of subjects, differences in diagnostic criteria of PA and the lateralization criteria of AVS rather than the impact of difference in imaging conditions of CT/MRI. However, as shown by Omura et al., patients with aldosterone-producing microadenomas could be diagnosed by AVS but not by imaging tests [22]. Therefore, whether AVS is necessary should be determined not only from the CT findings, but also the AST or other confirmatory tests such as the SIT.

In recent years, the number of cases with PA has increased because PA is widely known among not only endocrinologists but also general physicians. It has also become known that AVS is a very important test to determine localization in accordance with its wider spread. On the other hand, it is still far from easy for all PA patients to undergo AVS because of its negative 
aspects, which were described above. Therefore, bilateral cases of patients with PA should be identified in a minimally invasive manner before performing AVS.

There are several limitations in this study. While there have been several reports of discriminating between lateral and bilateral hypersecretion in PA using AST with dexamethasone $[12,13]$ and without dexamethasone [23], AST is still controversial in the diagnosis of PA. Next, previous studies reported varying prevalences of lateral lesions. Nishikawa et al. reported that among their 65 patients with PA, the proportions of APmacroA and APmicroA were $32.3 \%$ and $33.8 \%$, respectively, and APA predominated [6], while Young et al. reported that APA and IHA were distributed almost equally (APA, 53\%; IHA, 43\%) [26]. In the present study, cases of bilateral aldosterone excess such as IHA predominated. This difference in prevalence might be explainable by differences in diagnostic criteria for PA and the lateralization criteria of AVS. In general, the level of oversecretion in IHA is usually modest in comparison with that in APA. In the pres- ent study, using a high-sensitivity detection protocol by all confirmatory tests, the study group might have included cases that would be diagnosed as essential hypertension in other facilities. On the other hand, the advantage of the present study is that all cases underwent all confirmatory tests for diagnosis of PA (CCT, FUT, and SIT) and AST. There have been no previous reports about predictors for PA subtype in patients who underwent all confirmatory tests.

In conclusion, no tumor on $\mathrm{CT}$ scan and $\mathrm{PAC}_{\max } / \mathrm{F}$ less than 18.3 on the AST are useful for differentiating a unilateral lesion, such as APA, from a bilateral lesion such as IHA and the possibility that AVS might be less necessary. Thus, these findings suggest that abdominal CT and AST are useful for differentiating the subtype of PA and the indication for AVS.

\section{Disclosure}

None of the authors have any potential conflicts of interest associated with this research.

\section{References}

1. Millez P, Girerd X, Plouin PF, Blacher J, Safar ME, et al. (2005) Evidence for an increased rate of cardiovascular events in patients with primary aldosteronism. $J$ Am Coll Cardiol 45: 1243-1248.

2. Born-Frontsberg E, Reincke M, Rump LC, Hahner $\mathrm{S}$, Diederich S, et al. (2009) Cardiovascular and Cerebrovascular Comorbidities of Hypokalemic and Normokalemic Primary Aldosteronism: Results of the German Conn's Registry. J Clin Endocrinol Metab 94: 1125-1130.

3. Rossi GP, Bernini G, Desideri G, Fabris B, Ferri C, et al. (2006) Renal damage in primary aldosteronism: results of the PAPY Study. Hypertension 48: 232-238.

4. Rocha R, Stier CT Jr (2001) Pathophysiological effects of aldosterone in cardiovascular tissues. Trends Endocrinol Metab 12: 308-314.

5. Brown NJ (2003) Eplerenone: Cardiovascular Protection. Circulation 107: 2512-2518.

6. Nishikawa T, Saito J, Omura M (2007) Prevalence of Primary Aldosteronism: Should we screen for primary aldosteronism before treating hypertensive patients with medication? Endocr J 54: 487-495.

7. Nishikawa T, Omura M, Satoh F, Shibata H, Takahashi $\mathrm{K}$, et al. (2011) The Japan Endocrine Society Guidelines for the diagnosis and treatment of primary aldosteronism--the Japan Endocrine Society 2009. Endocr J 58: 711-721.
8. Shimamoto K, Ando K, Fujita T, Hasebe N, Higaki J, et al. (2014) The Japanese Society of Hypertension Guideline for the Management of Hypertension (JSH 2014). Hypertens Res 37: 253-390.

9. Omura M, Nishikawa T (2006) Screening tests and diagnostic examinations of hypertensives for primary aldosteronism. Rinsyo Byori 54: 1157-1163 (In Japanese).

10. Youg WF (2007) Primary aldosteronism: renaissance of a syndrome. Clin Endocrinol (Oxf) 66: 607-618.

11. Magill SB, Raff H, Shaker JL, Brickner RC, Knechtges TE, et al. (2001) Comparison of adrenal vein sampling and computed tomography in the differentiation of primary aldosteronism. J Clin Endocrinol Metab 86: 1066-1071.

12. Sonoyama $\mathrm{T}$, Sone $\mathrm{M}$, Miyashita $\mathrm{K}$, Tamura $\mathrm{N}$, Yamahara K, et al. (2011) Significance of adrenocorticotropin stimulation test in the diagnosis of an aldosterone-producing adenoma. J Clin Endocrinol Metab 96: 2771-2778.

13. Jiang Y, Zhang C, Wang W, Su T, Zhou W, et al. (2015) Diagnostic value of ACTH stimulation test in determining the subtypes of primary aldosteronism. J Clin Endocrinol Metab 100: 1837-1844.

14. Hiramatsu K, Yamada T, Yukimura Y, Komiya I, Ichikawa K, et al. (1981) A screening test to identify aldosterone-producing adenoma by measuring plasma renin activity: Results in hypertensive patients. Arch 
Intern Med 141: 1589-1593.

15. Onozawa S, Murata S, Tajima H, Yamaguchi H, Mine T, et al. (2014) Evaluation of right adrenal vein cannulation by computed tomography angiography in 140 consecutive patients undergoing adrenal venous sampling. Eur J Endocrinol 170: 601-608.

16. Satoh F, Abe T, Tanemoto M, Nakamura M, Abe M, et al. (2007) Localization of aldosterone-producing adrenocortical adenomas: significance of adrenal venous sampling. Hypertens Res 30: 1083-1095.

17. Küpers EM, Amar L, Raynaud A, Ploulin PF, Steichen O (2012) A clinical prediction score to diagnose unilateral primary aldosteronism. J Clin Endocrinol Metab 97: 3530-3537.

18. Nanba K, Tsuiki M, Nakao K, Nanba A, Usui T, et al. (2014) A subtype prediction score for primary aldosteronism. J Hum Hypertens 28: 716-720.

19. Minami I, Yoshimoto T, Hirono Y, Izumiyama H, Doi M, et al. (2008) Diagnostic accuracy of adrenal venous sampling in comparison with other parameters in primary aldosteronism. Endocr J 55: 839-846.

20. Rossi GP, Belfiore A, Bernini G, Desideri G, Fabris B, et al. (2007) Comparison of the captopril and the saline infusion test for excluding aldosterone-producing adenoma. Hypertension 50: 424-431.

21. Stowasser M, Klemm SA, Tunny TJ, Gordon RD (1995) Plasma aldosterone response to ACTH in sub- types of primary aldosteronism. Clin Exp Pharmacol Physiol 22: 460-462.

22. Omura M, Sasano H, Saito J, Yamaguchi K, Kakuta Y, et al. (2006) Clinical characteristics of aldosterone-producing microadenoma, macroadenoma, and idiopathic hyperaldosteronism in 93 patients with primary aldosteronism. Hypertens Res 29: 883-889.

23. Terui K, Kageyama K, Nigawara T, Moriyama T, Sakihara S, et al. (2016) Evaluation of the (1-24) adrenocorticotropin stimulation test for the diagnosis of primary aldosteronism. J Renin Angiotensin Aldosterone Syst 17: 1470320315625703.

24. Hirohara D, Nomura K, Okamoto T, Ujihara M, Takano K (2001) Performance of the basal aldosterone to renin ratio and of the renin stimulation test by furosemide and upright posture in screening for aldosterone-producing adenoma in low renin hypertensives. J Clin Endocrinol Metab 86: 4292-4298.

25. Kempers MJ, Lenders JW, van Outheusden L, van der Wilt GJ, Schultze Kool LJ, et al. (2009) Systematic Review: Diagnostic Procedures to Differentiate Unilateral From Bilateral Adrenal Abnormality in Primary Aldosteronism. Ann Intern Med 151: 329-337.

26. Young WF, Stanson AW, Thompson GB, Grant CS, Farley DR, et al. (2004) Role for adrenal venous sampling in primary aldosteronism. Surgery 136: 1227-1235. 